\title{
Study on Evaluation of Machine Learning Approaches in Brain Tumour MR Images
}

\author{
Nisha Josepha, D. Murugan ${ }^{b}$, A. Ramya ${ }^{c}$, S. Vijayakumard, T. Ganesh Kumar \\ aResearch Scholar, Department of Computer Science and Engineering, Manonmaniam Sundaranar University, \\ Abishekapatti, Tirunelveli, India. E-mail: nishajoseph226@gmail.com \\ ${ }^{\text {b} P r o f e s s o r ~ \&, ~ H O D, ~ D e p a r t m e n t ~ o f ~ C o m p u t e r ~ S c i e n c e ~ a n d ~ E n g i n e e r i n g, ~ M a n o n m a n i a m ~ S u n d a r a n a r ~ U n i v e r s i t y, ~}$ \\ Abishekapatti, Tirunelveli, India. E-mail: dhanushkodim@yahoo.com \\ cAssistant Professor, Department. of Computer Applications, B S Abdur Rahman Crescent Institute of S \&T, \\ India. E-mail: ramyaanandan10@gmail.com \\ ${ }^{\mathrm{d} R e s e a r c h ~ S c h o l a r, ~ D e p a r t m e n t . ~ o f ~ C o m p u t e r ~ A p p l i c a t i o n s, ~ M a n o n m a n i a m ~ S u n d a r a n a r ~ U n i v e r s i t y, ~}$ \\ Abishekapatti, Tirunelveli, India. E-mail: svjaykumar@gmail.com \\ eAssistant Professor, School of Computing Sciences and Engineering, Galgotias University, NCR, India. \\ E-mail: tganeshphd@yahoo.com
}

Article History: Received: 11 January 2021; Accepted: 27 February 2021; Published online: 5 April 2021

\begin{abstract}
The principal intention of this work is to compare the performance of the supervised brain tumour segmentation methods. These segmentation methods are based on machine learning. First, the input MR brain image is denoised by employing the adaptive bilateral filter, and the image contrast is enhanced employing the histogram equalization. Then we retrieve the features from the pre-processed image. Among several feature extraction methods, this work uses the shape, intensity, and texture feature extractors. Subsequent to removing these three types of features, fragment the tumor dependent on these recovered segments. The supervised segmentation approach is used for this. Among several supervised segmentation methods, this work uses three machine learning methods, namely Probabilistic Neural Network (PNN), Artificial Neural Network (ANN), and Convolution Neural Network (CNN). Finally, the retrieved features are feed into these machine learning methods to segment the brain tumour regions. To find out the best machine learning approach, the performance of these three supervised machines learning methods is evaluated by four performance metrics. Based on these evaluations, the best segmentation approach is discovered. Four execution boundaries are utilized, in particular, Dice Similarity Coefficient (DSC), Positive Predictive Value (PPV), Jaccard list (JI), and Sensitivity (SEN) to analyze the presentation of the AI strategy. The experimental outputs exposed that the CNN makes greater than other methods.
\end{abstract}

Keywords: MRI, Brain Tumour, Fully Automatic, Machine Learning Approach, PNN, ANN and CNN.

\section{Introduction}

Abnormal tissues in the brain cause brain tumours. The brain tumour is anomalous and non-controllable cell proliferation. Some occur in the brain on their own. This type of tumours is called preliminary. The one that extends to this place from wherever besides in the body in the spread and is called the second. Both are potentially lethal and life-threatening. Fast and rapid brain tumour diagnosis is the key to successful treatment. Here image acts as an essential role in the early diagnosis of the brain tumour. The most common way to discover tumours is CT and MRI [1]. The MRI is better because of its high resolution, especially in brain images. Besides, MRI images are not based on iodine radiation, so it is safer. Image sharing is a momentous stage in the investigation of medical images.

The brain image's main reason is to distribute the region's exclusive image and the mutual exhaustion of the same, following predetermined criteria. On account of a tumor, the division is essential for the partition of the tumor tissue, the hard or the tumor, the edema, and the gray matter (GM), the white matter (WM), also cerebrospinal liquid (CSF). The piece of the tumor needs focused on boundaries to decide the closeness, everything being equal. There are two ways to get a measure of unmasking and oversight.

Researchers divide the approaches for segmenting brain tumours into manual partition, semiautomatic partition, and fully automatic partition. Manual brain tumours partition involves the manual drawing of the tumour's boundary and the structure of interest or the patient's appearance on the anatomical structure's surface with a different label. Nevertheless, the physical division is too extensively employed in medical experiments, mainly when there is a lot of people's knowledge and attempts to identify the tissue. In the semi-autonomic part of the brain tumour, we often need human interventions to initiate a method of verifying the results or adjusting the distribution results. The semiautomatic part makes use of different schemes to integrate computers and human skills. The results of those ways rely upon ways and calculations. A half-automatic distribution like 
manual turnover will vary between specialists and users alike. The manual method of division takes a lot of time, and it can take about 3 hours to complete. The time taken is the key barrier to this approach.

In a fully automatic method, computers determine the part of the tumour without human interaction. Automated methods fully include person cleverness and advance information in techniques and are commonly created employing soft calculations in addition to model-based methods such as possible models. The fully automated fragmentation mode currently entirely desirable when processing multiple layouts. Automated segments can increase the doctor's ability and reduce the time needed for a proper diagnosis. The Researchers separate an autonomic segmentation method into two groups. They are Supervised and Unsupervised.

The main limitation of the unsupervised segmentation methods is the number of areas that often need to be determined. The tumour can spread to several parts, and the tumour may not have the intensity or the delimiter. The method for image segments differs from non-preserved ways of employing labelled learning data. The surveyed class includes training sessions that use tagged data to learn a particular model, a label, and a phase test used to label unbalanced data depending on estimated functions. In the controlled areas, selecting specific academic data is essential since different study groups guided many differences during the study and the difference between the distribution result. The manner that governs the operator depends on the collected scan data and the number of tissue cells in the image. The main benefit of employing a supervised approach is that the monitored method can dissimilar works only by altering the trainers' set. The noted method can reduce engineering tasks by giving data labelled with suitable features and suitable parameters for the learning algorithm.

This work's primary purpose is to segment the brain tumour employing MR images to provide medical assistant. The objective can be classified into the following tasks:

1. implementation of existing methods such as segmentation and feature extraction methods.

2. Studying the limitations and advantages of these implemented methods and

3. discover the best way for developing a new automated technique for the segmentation and feature extraction of segmenting the brain tumour from MRI.

We have summarised the paper in the following manner. In part 2, we have given a brief literature review. Part 3 shows a short report of the pre-measure system, division, and feature extraction method. This segment likewise displays the total stream outline of this proposed work. Part 4 clarifies the arrangement of information utilized for assessment of the performance and metrics utilized for this assessment. At last, we portray the completion of this research toward the end.

\section{Background}

There are several methods that exists for segmenting the brain tumour from MRI. Among them, this paper discussed a few of those methods. Bhagwat et al. [2] offer a paper showing the cluster method's evaluation, which means the K-mean, Fuzzy C, and Hierarchical mean for tumour discovery. MR brain images in nonmedical formats (.jpg,.png,.bmp, etc.) and digital images and medical contacts (DICOM) Image helps in experiencing the K-mean triangle, the FCM, and the hierarchy. DICOM images have been confirmed to produce better results for non-medical imaging. Suppose hierarchical partitioning is the smallest and fuzzy. In that case, it is maximal to identify the brain tumour where the K-agent method gives an additional exact output than Fuzzy cagents and hierarchical partitioning. Brain tumour discovery occupies various phases, for instance, image pretreatment, extraction of features, partition, and recognition. Laddha et al. [3] describe brain tumour paper employing the preconditioning process of normalization, and enhancement methods are used to increase the image's contrast. Image segmentation is then performed by segmentation of the threshold, segmentation of the catchment layer, and morphological techniques.

Madhikar et al. [4] revealed the tumour discovered in the brain by extracting image characteristics from MRI. The magnetic processing of high-quality medical imaging. SivaSankari et al. [6] Use the Gray-Level Cooccurrence Matrix (GLCM) to derive their processing features. PushpaRathi and others. [7] Their work found muscle discovery, which was made by step of the image, normalize, intensity, shape and texture extraction, selection of assortments, and assortment. Islam et al. [8]. This work provides a new collection of multiple Fractal Extracts (MultiFD) and controlled classification techniques for the surgery and measurement of the part of the brain tumour. MultiFD's features show the complex structure of tumour tissue in the brain's MRI, a multicellular transformation process in the brain's MRI. Charutha et al. [9] Brain tumour screening combine modified textile areas and elevations in the cellular system. Deepthi Murthy et al. [10] have exposed a method to get images of 
image abuse employing sound filters, image enhancements through histogram alignment, vector separation and morphological processing, and tumour discovery.

Alfonse and Salem [11] demonstrate techniques for the programmed metabolism of MR tumours employing an SVM measuring device. To increase the classification accuracy, the researchers removed FFT traits, and the trademark decrease was done utilizing the Minimal Redundancy-Maximal-Relevance (MRMR) procedure. In recent years, the interest in instrument preparation for the diagnosis of the tumour has increased. Gopal and Carney [12] used algorithms to assemble group image-shaped images with brain tumours and other non-existent groups. A set of data used in this work contains images with the MRI 42 from the KGB database. During the preliminary stages, the film's author, the X-labeled artifact, is available. They furthermore employ a metal filter to eliminate significant occurrence in formation in brain images. The author then uses a Cold Algorithm C, which means (FCM) cluster algorithm as a picture, and employs the genealogy method (GA) for optimizing purposes.

To correctness the truthfulness, the publisher employed a selection approach named Particle Swarm Optimization (PSO). The publishers handled this approach to attain a high correctness stage. At last, Najadat et al. [13] invent a recognizer to identify abnormalities in imagery reason for several diseases. Adel Kermi et al. [26] proposed an altogether automated, snappy, and exact mind tumor division strategy that naturally identifies and extricates tumors from MRI. This technique depends on a blend of three strategies brain symmetry analysis, locale based, and limit-based segmentation. The tests indicated that the localization and division results were good, albeit the strategy can be additionally improved. It is apparent that the odds of endurance of a tumorcontaminated patient can be expanded altogether if the tumor is distinguished precisely in its beginning phase (i.e., small tumor size). The kernel-based CNN method uses MRIs and M-SVM to classify the tumour segmented by kernel-based CNN [29]. Differential Evolution algorithms optimize the particular image's threshold value and train the neural network for brain tumour segmentation [30]. Munendra Singh et al. [27] proposes the use of dynamic contrast enhancement of MRI. It is even possible to detect very small benign but endocrinologically significant tumours called microadenoma. Such type of lesions is tough to detect even after checking of intravenous gadolinium. Guoqing $\mathrm{Wu}$ et al. [28] propose a sparse representation-based radio mics (SRR) framework to diagnose brain tumours. The inadequate portrayal-based component also known as SRR determination technique is adapted to choose a couple of critical highlights, but it takes a long effort to process.

\section{Materials and Methods}

The general design for the brain tumor division based managed learning has been exposed in diagram 1 below. To begin with, the information brain MRI is denoised by utilizing the versatile bilateral channel, and afterward the picture contrast is upgraded by utilizing the histogram equalization.

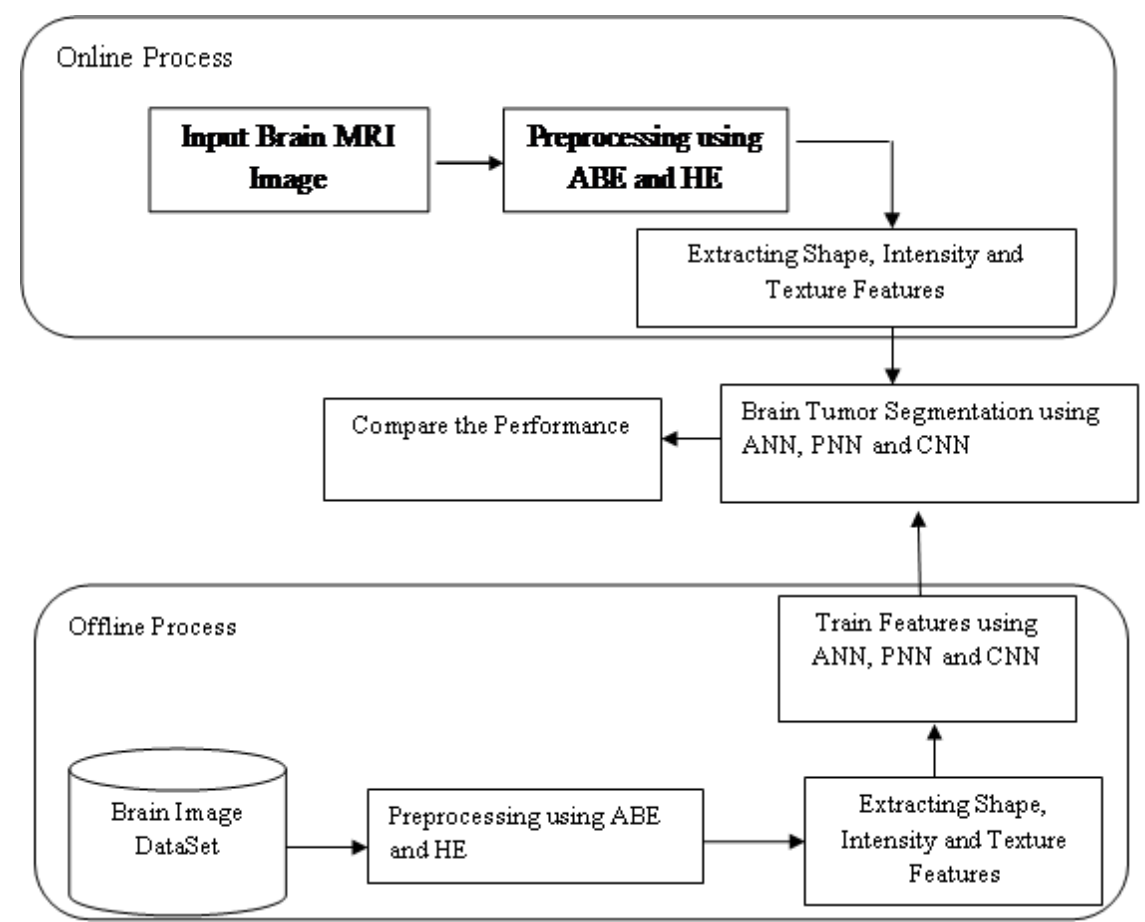

Figure 1. Overall Architecture of the Proposed Work 
After pre-processed the input image, the next stage is to retrieve the features from the denoised and contrastenhanced image. Among a lot of element extraction strategies, this work utilizes the shape, intensity, and texture feature extractors. Subsequent to extricating these three types of highlights, the following stage is to segment tumor dependent on these recovered highlights. The supervised segmentation approach is used for this process. Among a few segmentation strategies, this research utilizes three AI techniques: PNN, ANN, and CNN. At last, the recovered highlights are given as contribution to these AI strategies to section the tumor locales. To find out the best machine learning approach, the performance of these three supervised machines learning methods is evaluated by four performance metrics. Based on these evaluations, the best segmentation approach is discovered.

\subsection{Pre-Processing}

Initially, for preparation, the difference of info MR brain pictures is enhanced by utilizing the Histogram Equalization (HE) method. The improved pictures are portrayed in Figure 2 below.
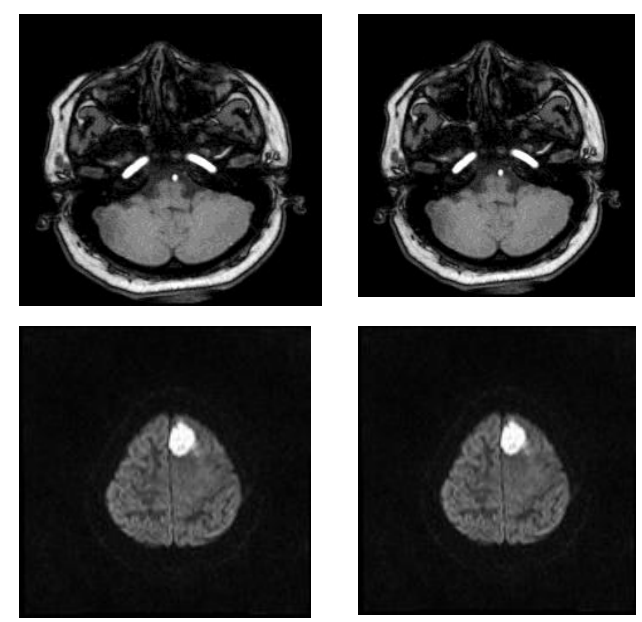

(a)

(b)

Figure 2. (a). Input MR Brain Image, (b). The Upgraded Picture Employing Histogram Equalization

Next, for pre-processing, the Adaptive Bilateral Filter (ABF) enhances brain MRI for de-noising. The denoised images are depicted in Fig. 3.

$$
\delta\left[x_{0,}, y_{0}\right]=\left\{\begin{array}{c}
\operatorname{MAXIMUM}\left(\beta_{x_{0}, y_{0}}\right)-G\left[x_{0,} y_{0}\right], \text { if } \Omega_{x_{0}, y_{0}}>0 \\
\operatorname{MINIMUM}\left(\beta_{x_{0} y_{0}}\right)-G\left[x_{0}, y_{0}\right], \text { if } \Omega_{x_{0} y_{0}}<0 \\
0, \quad \text { if } \Omega_{x_{0, y_{0}}}=0
\end{array}\right.
$$
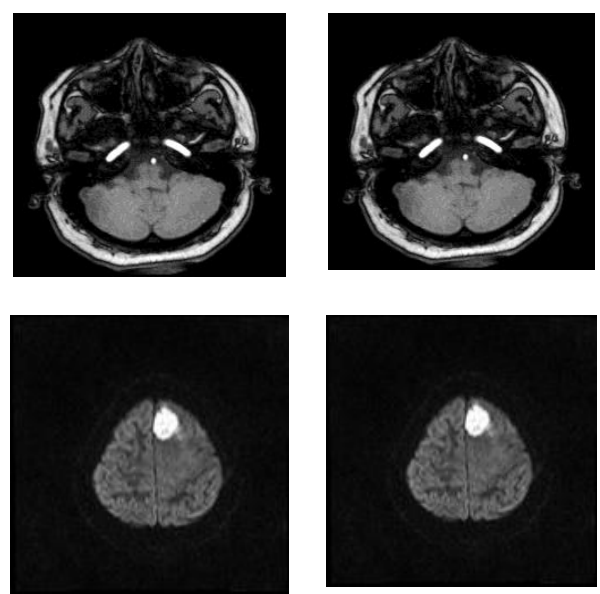

(a)

(b)

Figure 3. (a). Histogram Equalised Image, (b). Bilateral Filtered Image 


\subsection{Feature Extraction}

After de-noising and difference upgrade measure, the following stage is to recover the highlights from the processed pictures. This stage extracts three types of feature from each input image. The three types of features are listed below.

1. Shape -Perimeter, Area, circularity, irregularity, Shape Index

2. Intensity - Standard Variance, Mean, Median, Kurtosis, Variance, and Skewness

3. Texture -Correlation, Contrast, Homogeneity, Entropy, Cluster shade, Energy, the sum of square variance.

Therefore, three different types of features are retrieved to illustrates the organization data of intensity, shape, and texture. These features have a few redundancy numbers, excluding the reason for this step is to discover the brain tumour pixels through functional features.

\subsection{Classification}

After extracting the important element, the subsequent stage is to portion the cerebrum picture dependent on these recovered highlights utilizing a regulated segmentation approach. Among different directed division techniques, this work just concentrates the AI strategies. This work takes only three advanced machine learning methods from different machine learning methods, namely PNN, ANN, and CNN.

\subsubsection{Probabilistic Neural Network}

The probabilistic neural network is likely to be initiated by Donald Specht. This neural network depends on the Bayesian theory and estimation of probability density. It allows the characteristic value function to prove that it is worse than to classify the vector, which is a member of the class, and then arrange the class $\mathrm{n}$. The researcher examines this phase with magnetic resonance imaging (MRI). They examine it by employing the Image for MRI training and providing a sample of the tests attached to MRI. The scientists use it for segmentation. It is a sub-function of a feed-forward network obtained from the Bayesian one, and a statistical approach named kernel fisher discriminates analysis. For segmenting MRI, we employ the Bayes rule. We have illustrated the brain tumour partitioned images in Fig. 4, the non-brain tumour partitioned image in Fig.5, and the brain image in the border and its segmentation output in Fig.6.
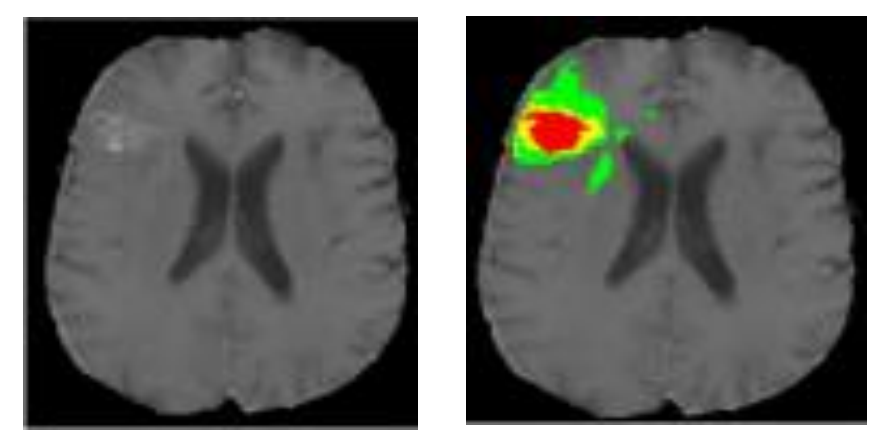

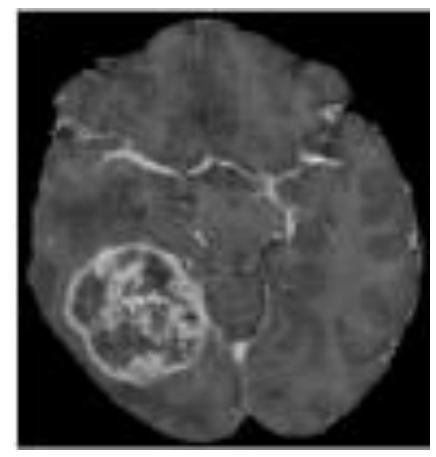

(a)

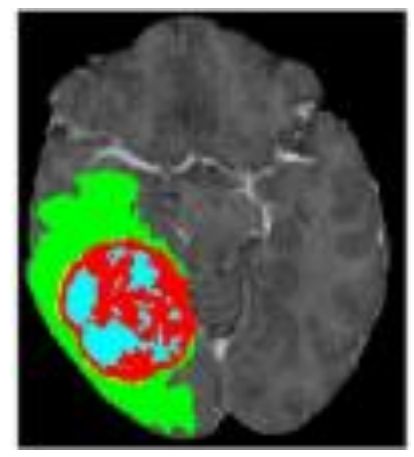

(b)

Figure 4. (a). Input Brain Image, (b). Segmented Image Employing PNN 

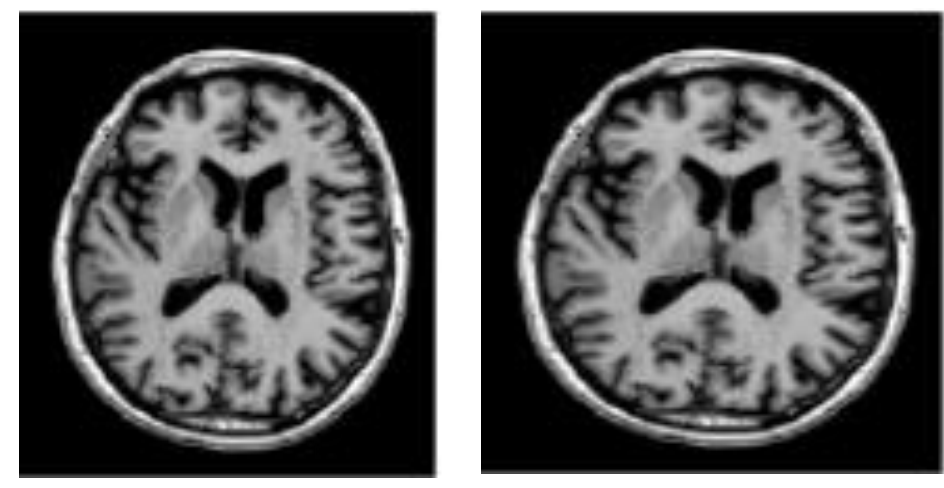

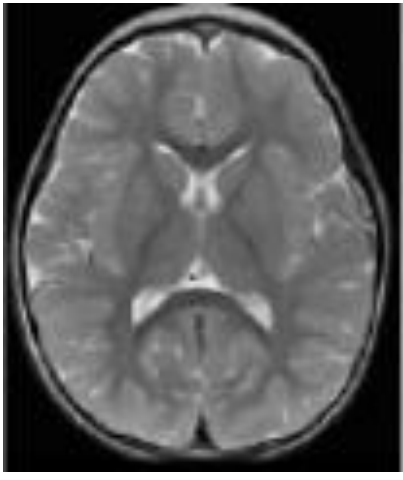

(a)

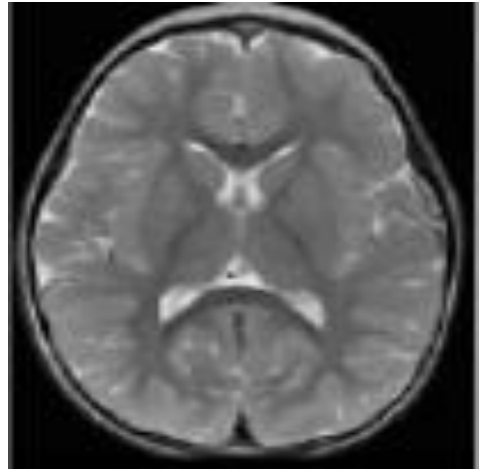

(b)

Figure 5. (a). Input Non Brain Image, (b). Segmented Image Employing PNN

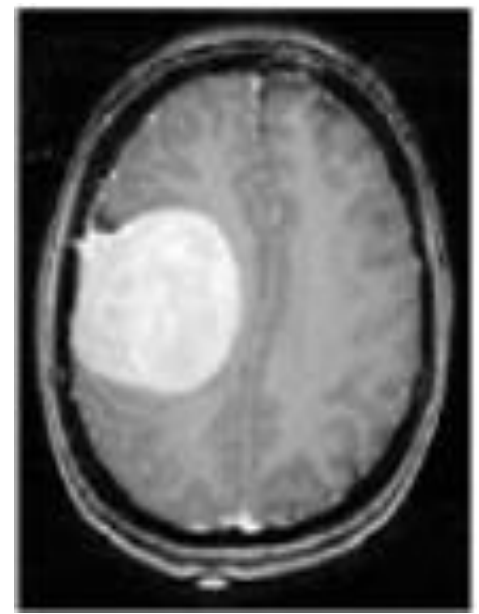

(a)

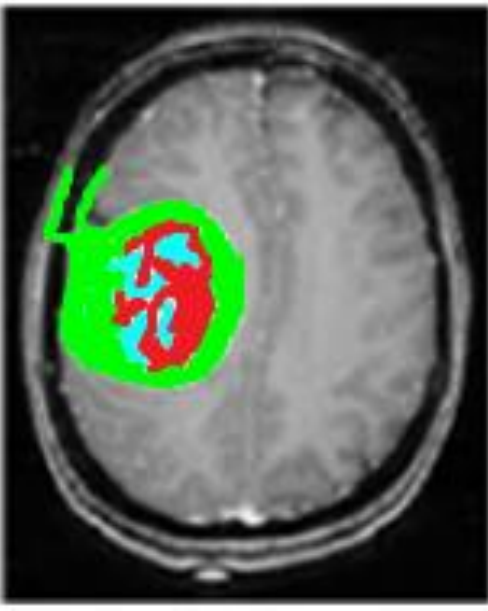

(b)

Figure 6. (a). Input Border Brain Image, (b). Segmented Image Employing PNN

\subsubsection{Artificial Neural Network}

An Artificial Neural Network [15-16] is one of the machine learning supervised segmentation approaches. Later than the training stage, the Artificial Neural Network variables are set as permanent, and the approach is installed to eliminate the limitation of segmenting the brain tumour. This network has one input layer, one or two hidden layers, and one output layer. By employing back-propagation, the retrieved features are continually given to the Artificial Neural Network. In every iteration, the neural network's result is evaluated to the brain tumour pixel values, and an error is estimated. This phase is called the training phase. The retrieved features are evaluated using the training values to determine the input image's brain tumour pixels in the testing stage. The brain tumour partitioned images are illustrated in Fig. 7, and the non-brain tumour partitioned image are illustrated in Fig. 8. The brain image in the border and its segmentation output is exposed in Fig.9 

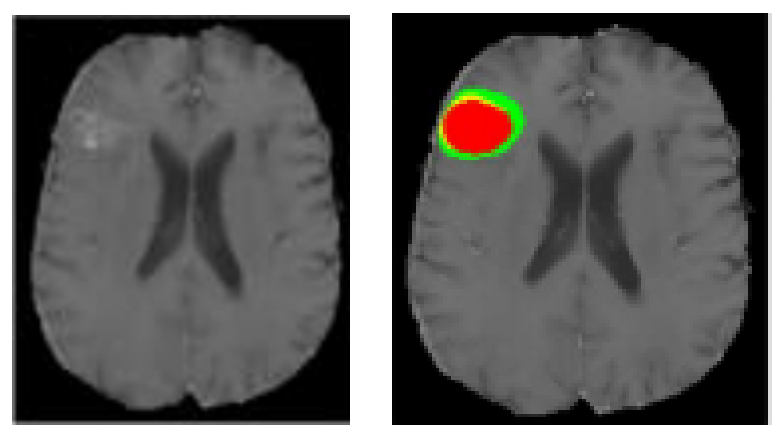

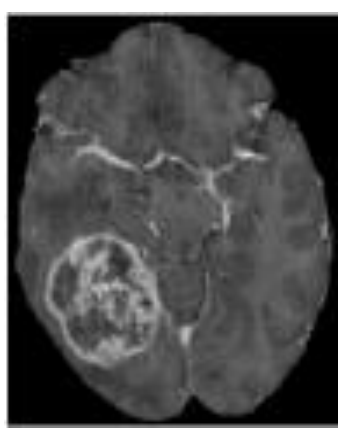

(a)

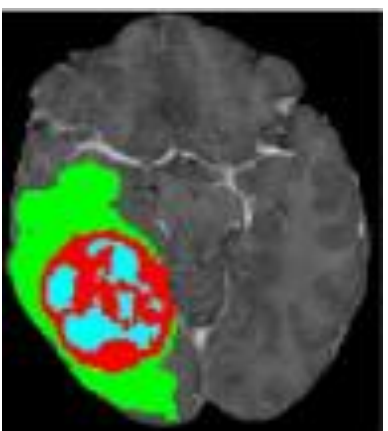

(b)

Figure 7. (a). Input Brain Image, (b). Segmented Image Employing ANN
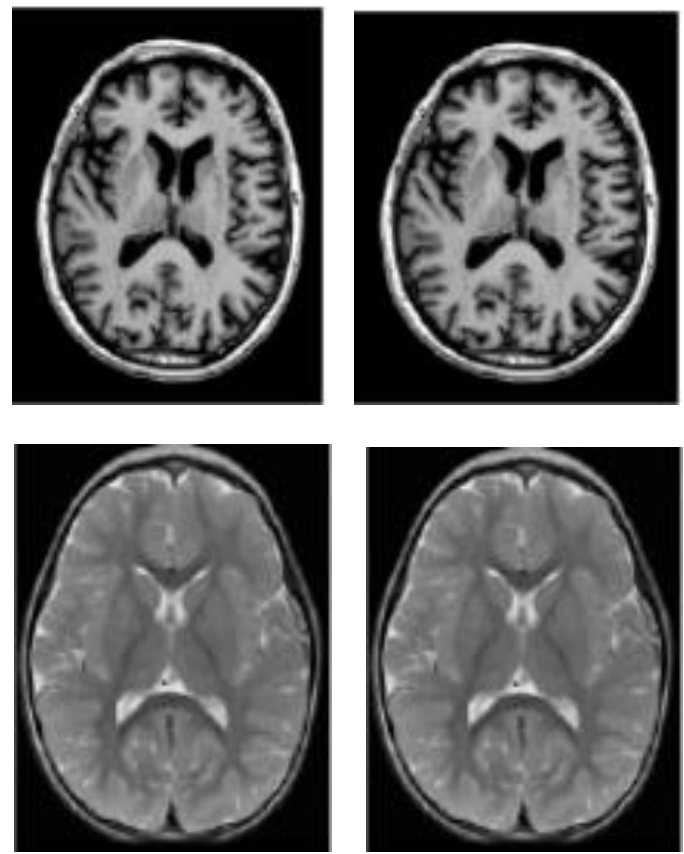

(a) (b)

Figure 8. (a). Input Non Brain Image, (b). Segmented Image Employing ANN

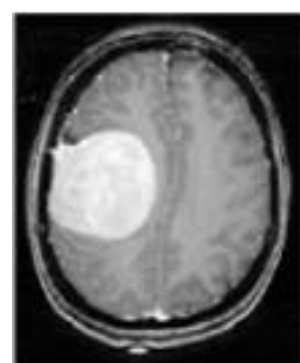

(a)

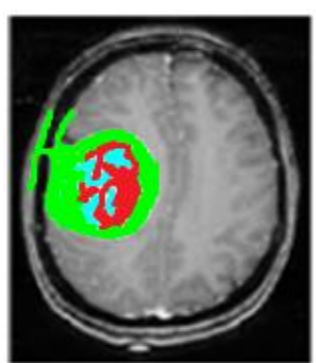

(b)

Figure 9. (a). Input Border Brain Image, (b). Segmented Image Employing ANN 


\subsubsection{Convolution Neural Network}

The CNN-based brain tumour segmentation [17-23] process has been separated into two training and testing stages. The quantity of pictures is segregated into various types, utilizing names, for example, tumors that are non-tumor brain pixels. In the CNN preparing stage, one segmentation model is developed on the retrieved features. CNN have three layers. They are Convolution, Pooling and Fully connected layer. In the convolution layer, the number of retrieved futures is increased by applying the convolution operation. The increasing the size of the features affects $\mathrm{CNN}$, so the pooling layer reduces the redundant feature values. Finally, the fully connected layer is employed to discover the brain tumour pixels employing the previously generated model. The brain tumour partitioned images are illustrated in Fig.a 10, and the non-brain tumour partitioned images are illustrated in Fig. 11. The brain image in the border and its segmentation output is exposed in Fig.12
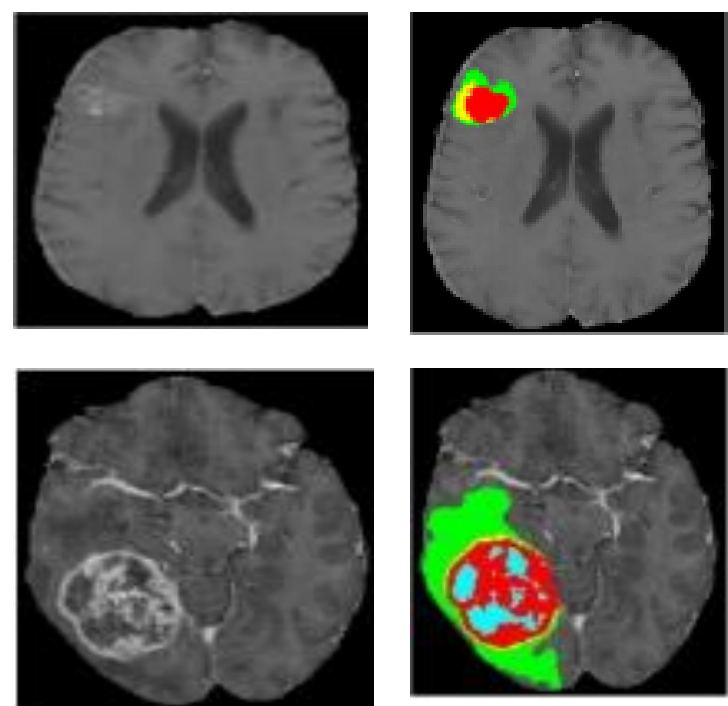

(a) (b)

Figure 10. (a). Input Brain Image,(b). Segmented Image Employing CNN
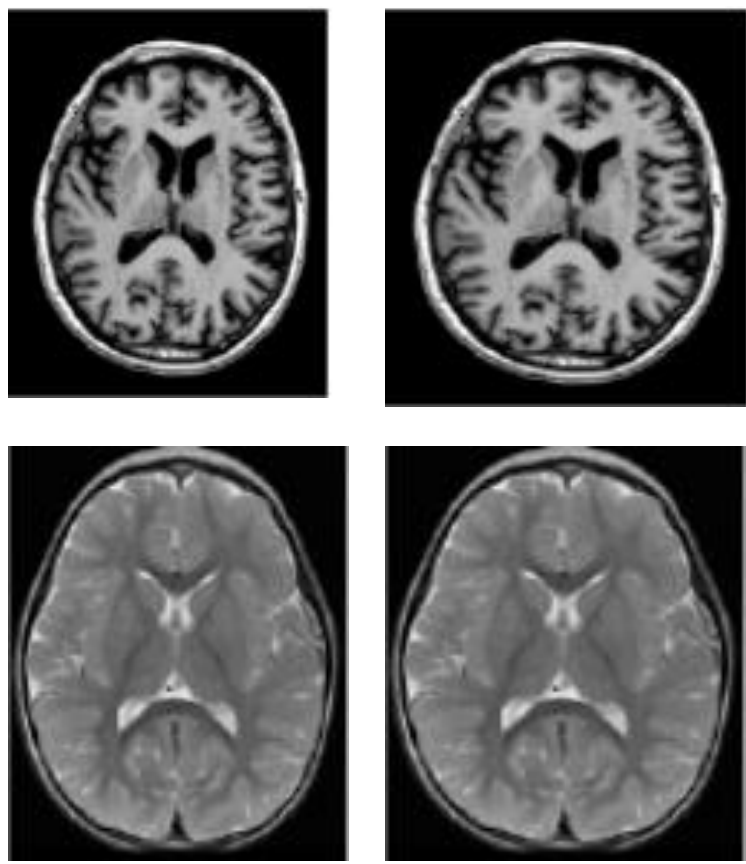

(a) (b)

Figure 11. (a). Input Non Brain Image, (b). Segmented Image Employing CNN 


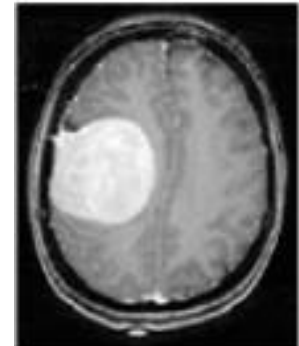

(a)

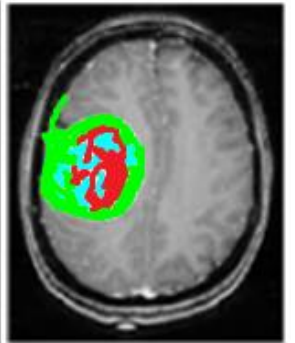

(b)

Figure 12. (a). Input Border Brain Image, (b). Segmented Image Employing CNN

\section{Experimental Results}

\subsection{DataSet Used}

We evaluated the machine learning methods presented in this paper on the BRATS 2015 databases.[24],[25]. Fig. 13 portrays the sampled tumor pictures from the BRATS 2015 data set.
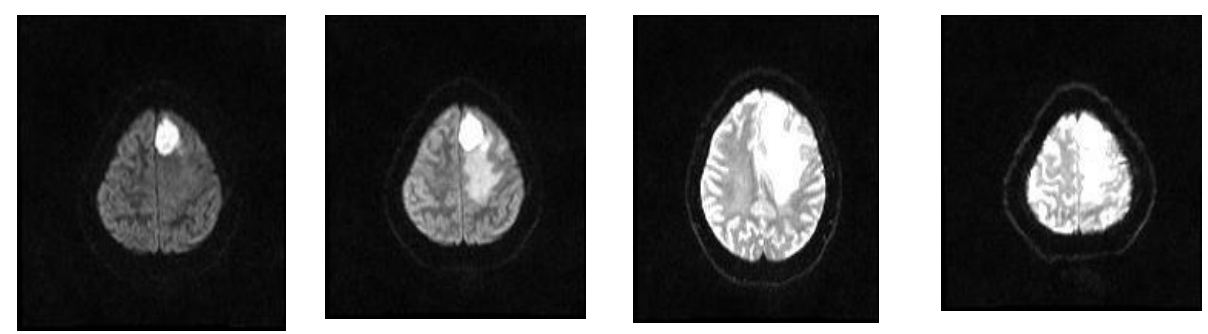

Figure 13. Experimental Images

\subsection{Performance Metric}

The appraisal of the administered AI division methods, this paper utilizes four measurements specifically Dice Similarity Coefficient (DSC), Positive Predictive Value (PPV), Jaccard index (JI), and Sensitivity (SEN).

\subsection{Experimental Study}

\subsubsection{Experiment No 1: Investigation of Feature Extraction Approaches on BRATS 2015 T1 Dataset}

This work will assess the involvement of each feature extraction method used in the analysis in this test. PPV, DSC, JI, and SEN parameters were employed to measure this efficiency feature extraction method. It is exposed in equations 4.5.6 and 7, respectively. Ideally, we expect a suitable feature extraction method to produce peak value for PPV, DSC, JI, and SEN. Table 1 lists the PPV, DSC, JI, and SEN parameters of the feature extraction method.

Table 1. Study of PPV, DSC, JI, and SEN of BRATS 2015 T1 Dataset for Feature Extraction Approaches

\begin{tabular}{lcccccccc}
\hline Metrics & DSC & PPV & JI & SEN & DSC & PPV & JI & SEN \\
\hline \multicolumn{9}{c}{ T1 } \\
\hline Shape & 82.273 & 91.173 & 94.393 & 93.813 & 80.923 & 89.823 & 93.043 & 92.463 \\
Texture & 83.183 & 92.193 & 93.563 & 94.903 & 81.833 & 90.843 & 92.213 & 93.553 \\
Intensity & 83.703 & 90.783 & 94.783 & 93.383 & 82.353 & 89.433 & 93.433 & 92.033 \\
All & $\mathbf{9 5 . 5 1 3}$ & $\mathbf{9 4 . 1 5 3}$ & $\mathbf{9 6 . 8 1 3}$ & $\mathbf{9 6 . 5 7 3}$ & 94.163 & 92.803 & 95.463 & 95.223 \\
\hline \multicolumn{8}{c}{ T1c } \\
\hline Shape & 82.453 & 91.353 & 94.573 & 93.993 & 83.693 & 92.593 & 95.813 & 95.233 \\
Texture & 83.363 & 92.373 & 93.743 & 95.083 & 84.603 & 93.613 & 94.983 & 96.323 \\
Intensity & 83.883 & 90.963 & 94.963 & 93.563 & 85.123 & 92.203 & 96.203 & 94.803 \\
All & 95.693 & 94.333 & 96.993 & 96.753 & 96.933 & 95.573 & 98.233 & 97.993 \\
\hline
\end{tabular}

As noted from Table 1, the PPV, DSC, JI, and SEN features are in limit 95-96, which is greater than that of the customary individual component extraction technique. In this way, the joined features are best for the tumor 
discovering approach. Diagram 8 portrayed the PPV, DSC, JI, and SEN boundaries of highlight extraction strategies.

\subsubsection{Experiment No 2: Study of Brain Tumour Segmentation Approaches on BRATS 2015 T1 Dataset}

In this test, this work will assess the involvement of each brain tumour partition method used in work. PPV, DSC, JI, and SEN parameters were employed to assess this brain tumour partition method's efficiency. It is exposed in equations 4.5.6 and 7, respectively. Ideally, a right brain tumour partition method is expected to produce peak value for PPV, DSC, JI, and SEN. Table 1 lists the PPV, DSC, JI and SEN parameters of the brain tumour partition method.

Table 2. Study of PPV, DSC, JI and SEN of BRATS 2015 T1 for Brain Tumour Segmentation Approaches

\begin{tabular}{lcccccccc}
\hline Metrics & DSC & PPV & JI & SEN & DSC & PPV & JI & SEN \\
\hline \multicolumn{9}{c}{ T1 } \\
\hline ANN & 84.412 & 93.422 & 94.792 & 96.132 & 83.202 & 92.212 & 93.582 & 94.922 \\
PNN & 84.932 & 92.012 & 96.012 & 94.612 & 83.722 & 90.802 & 94.802 & 93.402 \\
CNN & $\mathbf{9 6 . 7 4 2}$ & $\mathbf{9 5 . 3 8 2}$ & $\mathbf{9 8 . 0 4 2}$ & $\mathbf{9 7 . 8 0 2}$ & $\mathbf{9 5 . 5 3 2}$ & $\mathbf{9 4 . 1 7 2}$ & $\mathbf{9 6 . 8 3 2}$ & $\mathbf{9 6 . 5 9 2}$ \\
\hline \multicolumn{3}{c}{ T1c } & \multicolumn{5}{c}{ FLAIR } \\
\hline ANN & 82.262 & 91.272 & 92.642 & 93.982 & 83.412 & 92.422 & 93.792 & 95.132 \\
PNN & 82.782 & 89.862 & 93.862 & 92.462 & 83.932 & 91.012 & 95.012 & 93.612 \\
CNN & $\mathbf{9 4 . 5 9 2}$ & $\mathbf{9 3 . 2 3 2}$ & $\mathbf{9 5 . 8 9 2}$ & $\mathbf{9 5 . 6 5 2}$ & $\mathbf{9 5 . 7 4 2}$ & $\mathbf{9 4 . 3 8 2}$ & $\mathbf{9 7 . 0 4 2}$ & $\mathbf{9 6 . 8 0 2}$ \\
\hline
\end{tabular}

As noted from Table 2, the PPV, DSC, JI, and SEN of the CNN limit 96-97, which is greater than that of the other approach machine learning brain tumour segmentation. So, CNN is best for the brain tumour discovering approach.

\section{Conclusion}

Segmenting the brain tumour is confounded work so that the error can result in much more. This paper presented an extensive study comparing the supervised machine learning methods for brain tumour partitions. The performance of these methods is estimated by PPV, DICE, Sensitivity, and Jaccard. The experiments are done on BRATS 2015 dataset. This paper concluded that the CNN method produces the best result than PNN and ANN. Following DICE and Jaccard metrics, this paper justified that the CNN segmentation results were extraordinarily related to the ground truth segmentation. This paper intends to expand the number of training images and build up an efficient feature extraction method to enhance CNN's efficiency.

\section{References}

1. Bauer, S., Wiest, R., Nolte, L.P., \& Reyes, M. (2013). A survey of MRI-based medical image analysis for brain tumor studies. Physics in Medicine \& Biology, 58(13), 97-129.

2. Bhagwat, K., More, D., Shinde, S., Daga, A., \& Tornekar, R. (2013). Comparative study of brain tumor detection using $\mathrm{K}$-means, fuzzy $\mathrm{C}$ means and hierarchical clustering algorithms. International Journal of Engineering Science Res, 2, 626-632.

3. Laddha, R.R., \& Ladhake, S.A. (2014). A review on brain tumor detection using segmentation and threshold operations. International Journal of Computer Science and Information Technologies, 5(1), 607-611.

4. Madhikar, Ganesh., Sunita., \& Lokhande. S. (2013). Brain Detection and Classification by Using Modified Region Growing Method: A Review." International Journal of Engineering Research and Technology. 2(12).

5. Natarajan, P., Krishnan, N., Kenkre, N.S., Nancy, S., \& Singh, B.P. (2012). Tumor detection using threshold operation in MRI brain images. In IEEE International Conference on Computational Intelligence and Computing Research, 1-4.

6. SivaSankari, S., Sindhu, M., Sangeetha, R., \& ShenbagaRajan, A. (2014). Feature Extraction of Brain Tumour Using MRI, International Journal of Innovative Research in Science, Engineering and Technology, 3(3).

7. Demirhan, A., Törü, M., \& Güler, İ. (2014). Segmentation of tumor and edema along with healthy tissues of brain using wavelets and neural networks. IEEE journal of biomedical and health informatics, 19(4), 1451-1458.

8. Islam, A., Reza, S.M., \& Iftekharuddin, K.M. (2013). Multifractal texture estimation for detection and segmentation of brain tumors. IEEE transactions on biomedical engineering, 60(11), 3204-3215. 
9. Charutha, S., \& Jayashree, M.J. (2014, July). An efficient brain tumor detection by integrating modified texture based region growing and cellular automata edge detection. In International Conference on Control, Instrumentation, Communication and Computational Technologies (ICCICCT), 1193-1199.

10. Murthy, T.D., \& Sadashivappa, G. (2014). Brain tumor segmentation using thresholding, morphological operations and extraction of features of tumor. In International Conference on Advances in Electronics Computers and Communications, 1-6.

11. Alfonse, M., \& Salem, A.B.M. (2016). An automatic classification of brain tumours through MRI employing support vector machine, Egyptian Computer Science Journal, 40(3), 11-21.

12. Gopal, N.N., \& Karnan, M. (2010). Diagnose brain tumor through MRI using image processing clustering algorithms such as Fuzzy $\mathrm{C}$ Means along with intelligent optimization techniques. In IEEE International Conference on Computational Intelligence and Computing Research, 1-4.

13. Najadat, H., Jaffal, Y., Darwish, O., \& Yasser, N. (2011). A classifier to discover abnormality in CT brain images. In The IAENG International Conference on Data Mining and Applications, 374-377.

14. Zhang, B., \& Allebach, J.P. (2008). Adaptive bilateral filter for sharpness enhancement and noise removal. IEEE transactions on Image Processing, 17(5), 664-678.

15. Madhusudhanareddy, P., \& Prabha, I.S. (2013). Novel approach in brain tumor classification using artificial neural networks. International Journal of Engineering Research and Applications, 3(4).

16. Sonka, M., Hlavac, V., \& Boyle, R. (2004). Image processing, Study, and Machine Vision, II Edition, Vikas Publishing House, New Delhi, India.

17. Zikic, D., Ioannou, Y., Brown, M., \& Criminisi, A. (2014). Segmentation of brain tumor tissues with convolutional neural networks. Proceedings MICCAI-BRATS, 36, 36-39.

18. G. Urban et al., (2014). Multi-modal brain tumour segmentation employing deep convolutional neural networks, MICCAI Multimodal Brain Tumour Segmentation Challenge (BraTS), 1-5.

A. Davy et al., (2014). Brain tumour segmentation with deep neural networks, MICCAI Multimodal Brain Tumour Segmentation Challenge (BraTS), 31-35.

19. M. Havaei et al., (2015). Brain tumour segmentation with deep neural networks.

20. Lyksborg, M., Puonti, O., Agn, M., \& Larsen, R. (2015). An ensemble of 2D convolutional neural networks for tumor segmentation. In Scandinavian Conference on Image Analysis, 201-211.

21. Rao, V., Sarabi, M.S., \& Jaiswal, A. (2015). Brain tumor segmentation with deep learning. MICCAI Multimodal Brain Tumor Segmentation Challenge (BraTS), 56-59.

22. Menze, B.H., Jakab, A., Bauer, S., Kalpathy-Cramer, J., Farahani, K., Kirby, J., \& Van Leemput, K. (2014). The multimodal brain tumor image segmentation benchmark (BRATS). IEEE transactions on medical imaging, 34(10), 1993-2024.

23. Kistler, M., Bonaretti, S., Pfahrer, M., Niklaus, R., \& Büchler, P. (2013). The virtual skeleton database: an open access repository for biomedical research and collaboration. Journal of medical Internet research, 15(11), e245.

24. Dice, L.R. (1945). Measures of the amount of ecologic association between species. Ecology, 26(3), 297-302.

25. Kermi, A., Andjouh, K., \& Zidane, F. (2018). Fully automated brain tumour segmentation system in 3D-MRI using symmetry analysis of brain and level sets. IET Image Processing, 12(11), 1964-1971.

26. Singh, M., Verma, A., \& Sharma, N. (2017). Optimized multistable stochastic resonance for the enhancement of pituitary microadenoma in MRI. IEEE journal of biomedical and health informatics, 22(3), 862-873.

27. Wu, G., Chen, Y., Wang, Y., Yu, J., Lv, X., Ju, X., \& Chen, Z. (2017). Sparse representation-based radiomics for the diagnosis of brain tumors. IEEE transactions on medical imaging, 37(4), 893-905.

28. Thillaikkarasi, R., \& Saravanan, S. (2019). An enhancement of deep learning algorithm for brain tumor segmentation using kernel based CNN with M-SVM. Journal of medical systems, 43(4), 1-7.

29. Sharma, A., Kumar, S., \& Singh, S.N. (2019). Brain tumor segmentation using DE embedded OTSU method and neural network. Multidimensional Systems and Signal Processing, 30(3), 1263-1291.

30. Puja Shashi., \& Suchitra, R. (2019). Patch Based Deep Local Feature Learning and Self-Similarity Multi-Level Clustering for Neonatal Brain Segmentation in MR Images, International Journal of Recent Technology and Engineering, 9(2).

31. Nisha Joseph, Murugan, D., Basil John Thomas., \& Ramya A. (2020). Deep Weber Dominant Local Order Based Feature Generator and Improved Convolution Neural Network for Brain Tumour Segmentation in MR Images, International Journal of Engineering and Advanced Technology, 9(3). 